\title{
Stellar age dating with thorium, uranium and lead
}

\author{
Anna Frebel ${ }^{1}$ and Karl-Ludwig Kratz ${ }^{2}$ \\ ${ }^{1}$ McDonald Observatory and Department of Astronomy, University of Texas, \\ 1 University Station, C1402, Austin TX, 78712 \\ email: anna@astro.as.utexas.edu \\ ${ }^{2}$ Max-Planck-Institut für Chemie (Otto-Hahn-Institut), D-55128 Mainz, Germany \\ email: klk@uni-mainz.de
}

\begin{abstract}
We present HE 1523-0901, a metal-poor star in which the radioactive elements Th and U could be detected. Only three stars have measured U abundances, of which HE 1523-0901 has the most confidently determined value. From comparing the stable Eu, Os, and Ir abundances with measurements of Th and U, stellar ages can be derived. Based on seven such chronometer abundance ratios, the age of HE 1523-0901 was found to be 13 Gyr. Only an upper limit for $\mathrm{Pb}$ could be measured so far. Knowing all three abundances of $\mathrm{Th}, \mathrm{U}$, and $\mathrm{Pb}$ would provide a self-consistent test for $\mathrm{r}$-process calculations. $\mathrm{Pb}$ is the beta- plus alpha-decay end-product of all decay chains in the mass region between $\mathrm{Pb}$ and the onset of dominant spontaneous fission above $\mathrm{Th}$ and $\mathrm{U}$. Hence, in addition to $\mathrm{Th} / \mathrm{U}$ also $\mathrm{Th}, \mathrm{U} / \mathrm{Pb}$ should be used to obtain a consistent picture for actinide chronometry. From recent r-process calculations within the classical "waiting-point" model, for a 13 Gyr old star we predict the respective abundance ratios of $\log \epsilon(\mathrm{Th} / \mathrm{U})=0.84, \log \epsilon(\mathrm{Th} / \mathrm{Pb})=-1.32$ and $\log \epsilon(\mathrm{U} / \mathrm{Pb})=-2.16$. We compare these values with the measured abundance ratios in $\mathrm{HE} 1523-0901$ of $\log \epsilon(\mathrm{Th} / \mathrm{U})=0.86$, $\log \epsilon(\mathrm{Th} / \mathrm{Pb})>-1.0$ and $\log \epsilon(\mathrm{U} / \mathrm{Pb})>-1.9$. With this good level of agreement, HE 1523-0901 is already a vital probe for observational "near-field" cosmology by providing an independent lower limit for the age of the Universe.
\end{abstract}

Keywords. stars: abundances, stars: Population II, Galaxy: halo

\section{Introduction}

The first stars that formed from the pristine gas left after the Big Bang were very massive, of the order of $100 \mathrm{M}_{\odot}$ (Bromm et al. 2002). After a very short life time these so-called Population III stars exploded as supernovae, which then provided the first metals to the interstellar medium. All subsequent generations of stars formed from chemically enriched material. Metal-poor stars are early Population II objects and belong to the stellar generations that formed from the non-zero metallicity gas left behind by the first stars. Due to their low masses $\left(\sim 0.8 \mathrm{M}_{\odot}\right)$ they have extremely long life times that exceed the current age of the Universe of $\sim 14$ Gyr. Hence, these stellar "fossils" of the early Universe are still observable today. In their atmospheres these old objects preserve information about the chemical composition of their birth cloud and thus provide archaeological evidence of the earliest times of the Universe. In particular, the chemical patterns provide detailed information about the formation and evolution of the elements and the involved nucleosynthesis processes. This knowledge is invaluable for our understanding of the cosmic chemical evolution and the onset of star- and galaxy formation. Galactic metal-poor stars are the local equivalent of the high-redshift Universe. They allow us to derive observational constraints on the nature of the first stars and supernovae, and on various theoretical works on the early Universe in general (Frebel et al. 2007b). 
Focusing on long-lived low-mass metal-poor main-sequence and giant stars, we are observing stellar chemical abundances that reflect the composition of the interstellar medium during their star formation processes (Frebel et al. 2008). Stars spend $~ 90 \%$ of their life time on the main sequence before they evolve to become red giants. Mainsequence stars only have a shallow convection zone that preserves the stars' birth composition over billions of years. Stars on the red giant branch have deeper convection zones that lead to a successive mixing of the surface layers with nuclear burning products from the stellar interior. In the lesser evolved giants the surface composition has not yet been significantly altered by any such mixing processes and are useful as tracers of the chemical evolution. Further evolved stars (e.g., asymptotic giant branch stars) usually have surface compositions that have been altered through repeated events that dredge up events of nucleosynthetic burning products.

The main indicator used to determine stellar metallicity is the iron abundance, $[\mathrm{Fe} / \mathrm{H}]$, which is defined as $[\mathrm{A} / \mathrm{B}]=\log _{10}\left(N_{\mathrm{A}} / N_{\mathrm{B}}\right)_{\star}-\log _{10}\left(N_{\mathrm{A}} / N_{\mathrm{B}}\right) \odot$ for the number $\mathrm{N}$ of atoms of elements $\mathrm{A}$ and $\mathrm{B}$, and $\odot$ refers to the Sun. With few exceptions, $[\mathrm{Fe} / \mathrm{H}]$ traces the overall metallicity of the objects fairly well. This review focuses on metal-poor stars that have around 1/1,000 of the solar Fe abundances, and are thus able to probe the earliest epochs of nucleosynthesis processes. A summary of the history and the different "classes" of metal-poor stars and their role in the early Universe can be found in Frebel (2008) and an extensive review on the neutron-capture element abundances in (Sneden et al. 2008).

\section{Observing the r-Process signature in the oldest stars}

All elements except $\mathrm{H}$ and $\mathrm{He}$ are created in stars during stellar evolution and supernova explosions. About $5 \%$ of metal-poor stars with $[\mathrm{Fe} / \mathrm{H}]<-2.5$ contain a strong enhancement of neutron-capture elements associated with the rapid ( $\mathrm{r}-$ ) nucleosynthesis process (Beers \& Christlieb 2005) that is responsible for the production of the heaviest elements in the Universe. In those stars we can observe the majority (i.e., $\sim 70$ of 94 ) of elements in the periodic table: the light, $\alpha$, iron-peak, and light and heavy neutron-capture elements. These elements were not produced in the observed metal-poor star itself, but in a previous-generation supernova explosions. The so-called r-process metal-poor stars then formed from the material that was chemically enriched by such a supernova. This is schematically illustrated in Figure 1. We are thus able to study the "chemical fingerprint" of individual supernova explosions that occurred just prior to the formation of the observed star. So far, however, the nucleosynthesis site of the r-process has not yet unambiguously been identified, but supernovae with progenitor stars of $8-10 \mathrm{M}_{\odot}$ are the most promising locations (Qian \& Wasserburg 2003).

The giant HE 1523-0901 ( $V=11.1$ ) was found in a sample of bright metal-poor stars (Frebel et al. 2006) from the Hamburg/ESO Survey. It has the so far strongest enhancement in neutron-capture elements associated with the r-process $\dagger,[\mathrm{r} / \mathrm{Fe}]=1.8$. Its metallicity is $[\mathrm{Fe} / \mathrm{H}]=-3.0$ (Frebel et al. 2007a). The spectrum of HE 1523-0901 shows numerous strong lines of $\sim 25$ neutron-capture elements, such as those of $\mathrm{Sr}, \mathrm{Ba}, \mathrm{Eu}$, Os, and Ir. A full discussion of the complete abundance analysis will be given elsewhere (A. Frebel et al. 2009, in preparation). This makes possible a detailed study of the nucleosynthesis products of the r-process. This fortuitously also provides the opportunity of bringing together astrophysics and nuclear physics because these objects act as a "cosmic lab" for both fields of study.

$\dagger$ Stars with $[\mathrm{r} / \mathrm{Fe}]>1.0 ; \mathrm{r}$ represents the average abundance of elements from the r-process. 


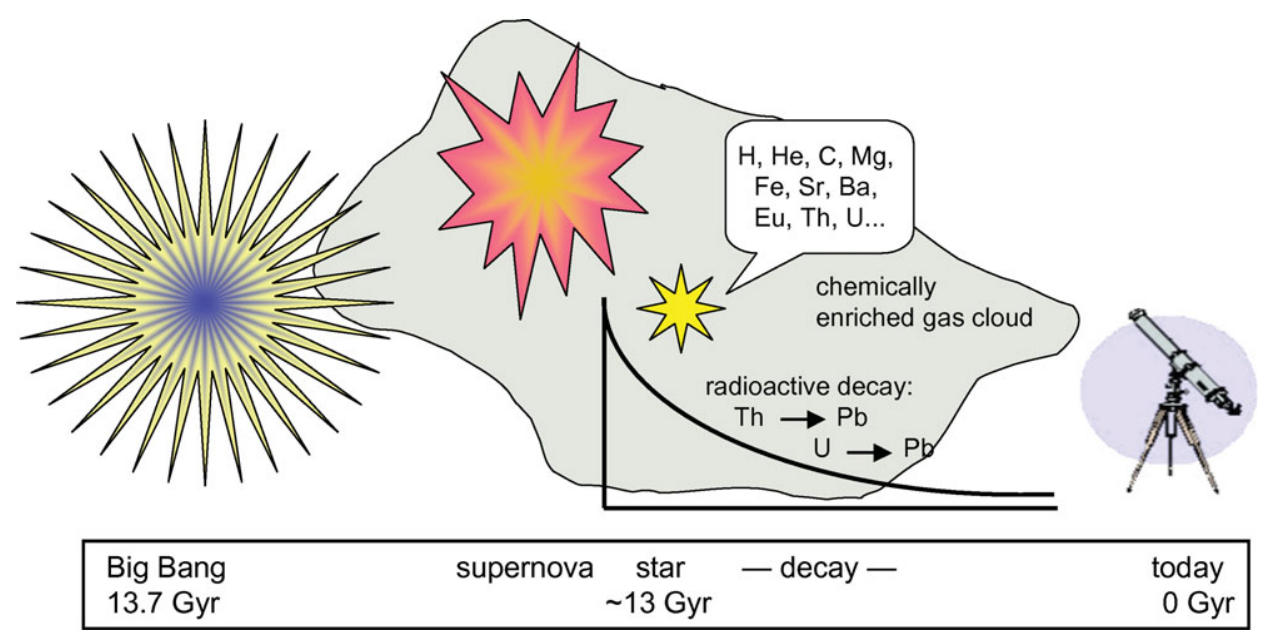

Figure 1. Formation process of r-process-enhanced metal-poor stars. They inherit the "chemical fingerprint" of a previous-generation supernova. Taken from Frebel (2009).

Although a rarity, HE 1523-0901 is not the only star that displays $[\mathrm{r} / \mathrm{Fe}]>1.5$. In 1995, the first r-process star was discovered, CS 22892-052 (Sneden et al. 1996) with $[\mathrm{r} / \mathrm{Fe}]=1.6$ and in 2001, CS 31082-001 (Cayrel et al. 2001) with the same overabundance in these elements. Their heavy neutron-capture elements follow the scaled solar r-process pattern, and offered the first vital clues to the universality of the r-process and the detailed study of the r-process by means of stars. As can be seen, in the mass range $56<Z<77$, the stellar abundances very closely follow the scaled solar r-process pattern Burris et al. (2000). This repeated behavior suggests that the r-process is universal - an important empirical finding that could not be obtained from any laboratory on earth. However, there are deviations among the lighter neutron-capture elements. It is not clear if the neutron-capture patterns are produced by a single r-process only, or if an additional new process might need to be invoked in order to explain all neutron-capture abundances.

\section{Nucleo-chronometry}

Among the heaviest elements are the long-lived radioactive isotopes ${ }^{232} \mathrm{Th}$ (half-life $14 \mathrm{Gyr})$ and ${ }^{238} \mathrm{U}(4.5 \mathrm{Gyr})$. While Th is often detectable in r-process stars, U poses a real challenge because only one, extremely weak, line is available in the optical spectrum. By comparing the abundances of the radioactive $\mathrm{Th}$ and/or $\mathrm{U}$ with those of stable $\mathrm{r}$ process nuclei, such as Eu, stellar ages can be derived. Through individual age measurements, r-process objects become vital probes for observational "near-field" cosmology. Importantly, it also confirms that metal-poor stars with similarly low Fe abundances and no excess in neutron-capture elements are similarly old, and that the commonly made assumption about the low mass $\left(0.6\right.$ to $\left.0.8 \mathrm{M}_{\odot}\right)$ of these survivors is well justified.

Most suitable for such age measurements are cool metal-poor giants that exhibit such strong overabundances of r-process elements. Since CS 22892-052 is very C-rich, however, the $\mathrm{U}$ line is blended and not detectable. Only the $\mathrm{Th} / \mathrm{Eu}$ ratio could be employed, and an age of $14 \mathrm{Gyr}$ was derived (Sneden et al. 2003). The U/Th chronometer was first measured in the giant CS 31082-001 (Cayrel et al. 2001) yielding an age of 14 Gyr. Since $\mathrm{Eu}$ and $\mathrm{Th}$ are much easier to detect than $\mathrm{U}$, the $\mathrm{Th} / \mathrm{Eu}$ chronometer is then used to derive stellar ages of r-process metal-poor stars. Compared to $\mathrm{Th} / \mathrm{Eu}$, the $\mathrm{Th} / \mathrm{U}$ ratio is much more robust to uncertainties in the theoretically derived production ratio due to 

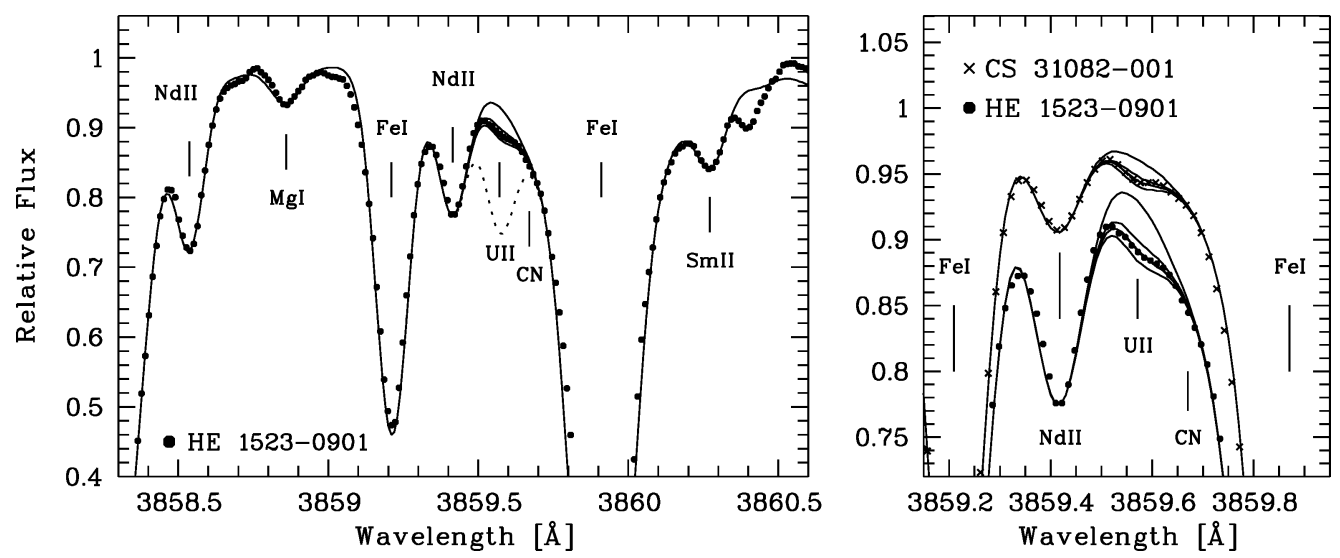

Figure 2. Spectral region around the U II line in HE 1523-0901 (filled dots) and CS 31082-001 (crosses; right panel only). Overplotted are synthetic spectra with different U abundances. The dotted line in the left panel corresponds to a scaled solar r-process $U$ abundance present in the star if no U were decayed. Figure taken from (Frebel et al. 2007a).

the similar atomic masses of Th and U (Wanajo et al. 2002), (Kratz et al. 2004). Hence, stars displaying Th and $\mathrm{U}$ are the most valuable old stars.

In addition to the heaviest stable elements in HE 1523-0901, also the radioactive isotopes Th and $\mathrm{U}$ were measured. In fact, the $\mathrm{U}$ measurement in this star is the currently most reliable one of the only three stars with such detections. Figure 2 shows the spectral region around the only available optical $U$ line from which the $U$ abundance was deduced. For HE 1523-0901, the availability of both the Th and U opened up the possibility for the first time to use seven different chronometer pairs consisting of Eu, Os, Ir, Th, and U. It should be noted, that the other star with a reliable U abundance, CS 31082-001, suffers from what has been termed an "actinide boost" (Honda et al. 2004). Compared with the scaled solar r-process (i.e., with other stable r-process elements), it contains too much Th and $\mathrm{U}$. Hence, its $\mathrm{Th} / \mathrm{Eu}$ ratio yields a negative age. The origin of this issue has yet to be understood. As a result, however, it has become clear that this star likely has a different origin (Kratz et al. 2007).

The averaged stellar age of HE 1523-0901 derived from seven chronometers $\mathrm{Th} / \mathrm{r}$, $\mathrm{U} / \mathrm{r}$ and $\mathrm{Th} / \mathrm{U}$ involving combinations of $\mathrm{Eu}, \mathrm{Os}, \mathrm{Ir}, \mathrm{Th}$ and $\mathrm{U}$ is $\sim 13 \mathrm{Gyr}$ (Frebel et al. 2007a). Such an age provides a lower limit to the age of the Galaxy and hence, the Universe which is currently assumed to be $13.7 \mathrm{Gyr}$ (Spergel et al. 2007). Unfortunately, realistic age uncertainties range from $\sim 2$ to $\sim 5$ Gyr. From a re-determination of the U/Th ratio in CS 31082-001 (Hill et al. 2002), a relative age of this star and HE 1523-0901 can be derived. HE 1523-0901 is found to be 1.5 Gyr younger than CS 31082-001, which is independent of the employed production ratio. This age difference is based on only a 0.07 dex difference in the observed U/Th ratios. Given that the observational uncertainties exceed that ratio difference, the derived ages of the two stars suggest that they formed at roughly the same time. This is also reflected in their almost identical metallicity of $[\mathrm{Fe} / \mathrm{H}] \sim-3.0$.

\section{At the end of everything: lead}

We also attempted to measure the $\mathrm{Pb}$ line at $4057 \AA$, the decay product of $\mathrm{Th}$ and U. However, it could not be detected in the current spectrum of HE 1523-0901 because the $S / N$ is not high enough. The upper limit of $\log \epsilon(\mathrm{Pb})<-0.2$ can be compared 
with predicted values for the total $\mathrm{Pb}$ abundance. $\mathrm{Pb}$ is produced through several decay channels. At the time when the r-process event stops, there is the $\operatorname{direct}(\beta$ - and $\beta$-delayed neutron) decay of very neutron-rich isobaric nuclei with $A=206-208$ to ${ }^{206} \mathrm{~Pb},{ }^{207} \mathrm{~Pb}$, and ${ }^{206} \mathrm{~Pb}$. Then there is $\alpha$ - and $\beta$-decay of nuclei with $A \geqslant 210$ back to $\mathrm{Pb}$ and finally the radioactive decay of the $\mathrm{Th}$ and $\mathrm{U}$ isotopes back to $\mathrm{Pb}$ over the course of the age of the Universe. The initial abundances of $\mathrm{Th}$ and $\mathrm{U}$ are driven in the same way as $\mathrm{Pb}$, i.e., by a direct channel of nuclei with $A=232,235$ and 238, and an indirect way from the decay of r-process nuclei with heavier masses. Of course, the Th and $\mathrm{U}$ abundances determine, in part, the $\mathrm{Pb}$ abundance. It is highly debated what the abundances of individual nuclei directly after an r-process really are. Hence, different models yielding different abundance distributions can be self-consistently constrained by explaining the stellar triumvirate of $\mathrm{Th}, \mathrm{U}$ and $\mathrm{Pb}$ abundances that are so intimately coupled not only with each other but also to the conditions (and potentially also the environment) of the r-process.

Following (Plez et al. 2004), we determine the $\mathrm{Pb}$ contribution from the decay of ${ }^{238} \mathrm{U}$ into ${ }^{206} \mathrm{~Pb},{ }^{232} \mathrm{U}$ into ${ }^{208} \mathrm{~Pb}$, and ${ }^{235} \mathrm{U}$ into ${ }^{207} \mathrm{~Pb}$, whereby the last one is based on a theoretically derived ratio of ${ }^{235} \mathrm{U} /{ }^{238} \mathrm{U}$. The total abundance of these three decays amounts to $\log \epsilon(\mathrm{Pb})=-0.72$. This is in agreement with our upper limit of $\log \epsilon(\mathrm{Pb})<-0.2$. It also leaves "room" for the direct and indirect decay channels that likely produce the main portion of the $\mathrm{Pb}$ in the star. Using r-process model calculations, predictions can then be derived for the total $\mathrm{Pb}$ to be measured in $\mathrm{HE}$ 1523-0901. Our r-process calculations are based on a site-independent model of the classical "waiting-point" approximation (see e.g., (Kratz et al. 1993), (Kratz et al. 2007)) using as nuclear physics input the ETFSI-Q mass model (Pearson et al. 1996) overlaid by experimental masses Audi et al. (2003) Audi, Wapstra, \& Thibault, and $\beta$-decay properties from QRPA calculations of Gamow-Teller and first-forbidden strength functions (Möller et al. 2003), again, where available, overlaid by experimental data (Pfeiffer et al. 2001).

After $13 \mathrm{Gyr}$ of decay, the prediction for the Th/U ratio in an old metal-poor star is $\log \epsilon(\mathrm{Th} / \mathrm{U})=0.84, \log \epsilon(\mathrm{Th} / \mathrm{Pb})=-1.32$ and $\log \epsilon(\mathrm{U} / \mathrm{Pb})=-2.16$. This agrees well with the values of $\log \epsilon(\mathrm{Th} / \mathrm{U})=0.86, \log \epsilon(\mathrm{Th} / \mathrm{Pb})>-1.0$ and $\log \epsilon(\mathrm{U} / \mathrm{Pb})>-1.9$ in HE 1523-0901. The value for Th/U also agrees well with $\epsilon(\mathrm{Th} / \mathrm{U})=0.89$ found in CS 31082-01. For $\mathrm{Pb}$ alone, our prediction of $\epsilon(\mathrm{Pb})=-0.346$ is also consistent with our current upper limit of $\log \epsilon(\mathrm{Pb})<-0.2$ in HE 1523-0901. With this good level of agreement, HE 1523-0901 is already a vital probe for observational "near-field" cosmology by providing an independent lower limit for the age of the Universe. However, our $\mathrm{Pb}$ prediction does not agree with the measured $\mathrm{Pb}$ abundance of $\log \epsilon(\mathrm{Pb})=-0.55$ in CS31082-001. The exact reasons for this discrepancy, in having a lower $\mathrm{Pb}$ abundance despite the actinide boost, are currently unknown and subject to much debate. The only possibility of resolving this problem, wile still upholding the assumption of a universal (main) r-process pattern in extremely metal-poor stars, would be to assume that at least part of the heavy r-elements including Th and U in CS31082-001 were implanted long after the formation of this star (Kratz et al. 2004). Overall, our calculations suggest that the $\mathrm{Pb}$ abundance in HE 1523-0901 may be high enough so that with new, higher $S / N$ data (500 or more at $4050 \AA$ ) a detection of the very weak Pb line at $4057 \AA$ should become feasible, or at least provide a much tighter and more constraining upper limit.

\section{Conclusion \& outlook}

Old metal-poor stars in our Galaxy have been shown to provide crucial observational clues to the nature of neutron-capture processes, in particular the r-process. However, even after dedicated searches, only about two dozens of these stars are known, and only 
three with any detection of U. Clearly, more such objects are needed to arrive at statistically meaningful abundance constraints for various r-process calculations. If more suitable candidates can be identified, and with large amounts of telescope time, more U as well as $\mathrm{Pb}$ abundances can be measured. Having all three measurements ( $\mathrm{Th}, \mathrm{U}$ and $\mathrm{Pb}$ ) available provides an excellent test bed for different r-process models because all three abundances have to be reproduced in a self-consistent manner. Such constraints, in turn, should lead to a better understanding of how and where r-process nucleosynthesis can occur. Improved r-abundance calculations are crucial for reliably predicting the initial production ratios of $\mathrm{Th} / \mathrm{r}, \mathrm{U} / \mathrm{r}$ and $\mathrm{Th} / \mathrm{U}$ and are implicitly required for a better age dating of $\mathrm{r}$-process enhanced stars. In the most optimistic (but probably not achievable) case of providing theoretical initial r-abundance ratios essentially free of systematic r-process model and nuclear-physics uncertainties, the deduced age uncertainties would then be reduced to the observational errors. In any case, better initial element production ratios will lead to improved age determinations, and with this will provide more meaningful lower limits of the age of the Universe, independent of and complementary to other methods. In this way, the possibility to carry out competitive "near-field" cosmology would strongly motivate our astronomer community to further search for search for the best and most suitable candidates for additional actinide measurements.

\section{Acknowledgements}

A. F. is supported through the W. J. McDonald Fellowship. K.-L. K. acknowledges partial support through the Deutsche Forschungsgemeinschaft under contract KR 806/13-1.

\section{References}

Audi, G., Wapstra, A. H., \& Thibault, C. 2003, Nuclear Physics A, 729, 337

Beers, T. C. \& Christlieb, N. 2005, ARAA, 43, 531

Bromm, V., Coppi, P. S., \& Larson, R. B. 2002, ApJ, 564, 23

Burris, D. L. et al. 2000, ApJ, 544, 302

Cayrel, R. et al. 2001, Nature, 409, 691

Frebel, A. 2009, subm. for PoS, Internatl. Symp. Nuclei in the Cosmos, Mackinaw Island, 2008

Frebel, A. 2008, in ASP Conference Series, Vol. 393, New Horizons in Astronomy, Frank N. Bash Symposium 2007, ed. A. Frebel, J. R. Maund, J. Shen, \& M. H. Siegel, 63

Frebel, A. et al. 2006, ApJ, 652, 1585

Frebel, A. et al. 2007a, ApJ, 660, L117

Frebel, A., Johnson, J. L., \& Bromm, V. 2007b, MNRAS, 380, L40

-. 2008, MNRAS, in press, astro-ph/0811.0020

Hill, V. et al. 2002, A\&A, 387, 560

Honda, S. et al. 2004, ApJ, 607, 474

Kratz, K.-L., Bitouzet, J.-P., Thielemann, F.-K., Moeller, P., \& Pfeiffer, B. 1993, ApJ, 403, 216

Kratz, K.-L. et al. 2007, ApJ, 662, 39

Kratz, K.-L., Pfeiffer, B., Cowan, J. J., \& Sneden, C. 2004, New Astronomy Review, 48, 105

Möller, P., Pfeiffer, B., \& Kratz, K.-L. 2003, Physics Review C, 67, 055802

Pearson, J. M., Nayak, R. C., \& Goriely, S. 1996, Physics Letters B, 387, 455

Pfeiffer, B., Kratz, K. ., \& Moeller, P. 2001, ArXiv Nuclear Experiment e-prints

Plez, B. et al. 2004, A\&A, 428, L9

Qian, Y.-Z. \& Wasserburg, G. J. 2003, ApJ, 588, 1099

Sneden, C., Cowan, J. J., \& Gallino, R. 2008, ARA\&A, 46, 241

Sneden, C. et al. 2003, ApJ, 591, 936

Sneden, C. et al. 1996, ApJ, 467, 819 
Spergel, D. N., et al. 2007, ApJS, 170, 377

Wanajo, S., Itoh, N., Ishimaru, Y., Nozawa, S., \& Beers, T. C. 2002, ApJ, 577, 853

\section{Discussion}

R. WYsE: As an advertisement for the RAVE survey, which is getting medium resolution spectra of $I<12$ stars, we are obtaining follow-up high-resolution spectra for candidate extremely- metal-poor stars. They are bright enough that 4-8 m telescopes suffice.

A. FREBEL: Any bright metal-poor stars would of course be extremely valuable. It would also tell us more about the spatial and kinematic distributions in the Galaxy since, generally, the more metal-poor stars are fainter.

J. VAlenti: 1) How many stars are waiting to be discovered that could be age-dated using thorium or uranium diagnostics? 2) Is a high-resolution ultraviolet spectrograph required for this type of analysis?

A. Frebel: 1) Most likely very few: bright r-process stars seem to be rare because otherwise we would have found them. Barklem et al. find $3-5 \%$ of stars with $[\mathrm{Fe} / \mathrm{H}]<$ -2.0 to be r- process rich. We are finding those $3-5 \%$, but they become fainter and fainter, making them inaccessible to high signal-to-noise spectroscopic observations.

I. RoEDERER: Regarding J. Valenti's question of needing STIS spectra, 1) all of the $\mathrm{Pb}$-normal measurements in stars with $[\mathrm{Fe} / \mathrm{H}]<-2.5$ are from the $\mathrm{Pb}$ I resonance line at $2833 \AA$ only. This is the only useful line in a number of stars in the regime of interest: old, metal-poor halo stars. 2) UV spectrographs are not required for identifying those stars, but they provide unprecedented information with regard to the overall chemical abundance pattern from which details about the r-process can be inferred.

I. ROEDERER: In the case where you have an ensemble of stars with measured thorium, and assuming that the stars are the same age and you just need to measure that age, this technique may hold great promise. It will be very difficult, I think, to improve upon the age uncertainties derived by Hill and Frebel for CS31082-001 and HE1523-0901, in the case of attempting to derive the age of a given field star using this technique. 


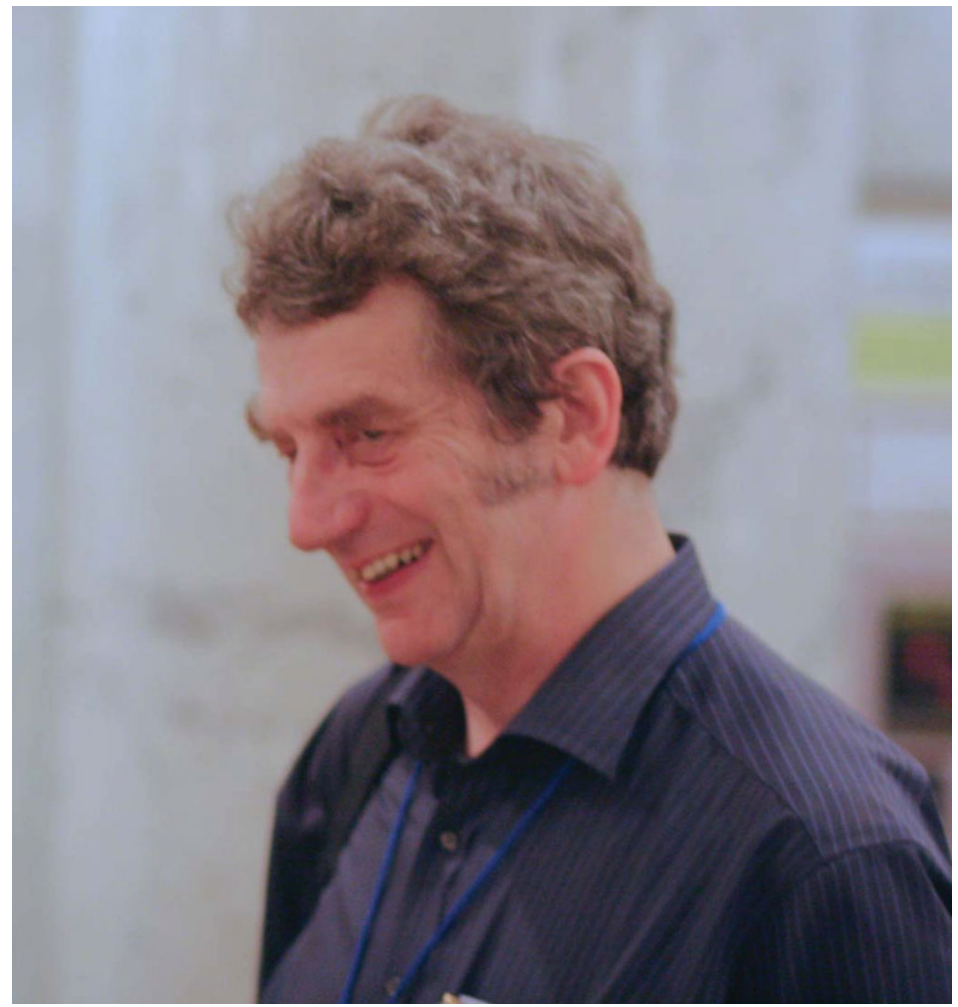

Joergen Christensen-Dalsgaard

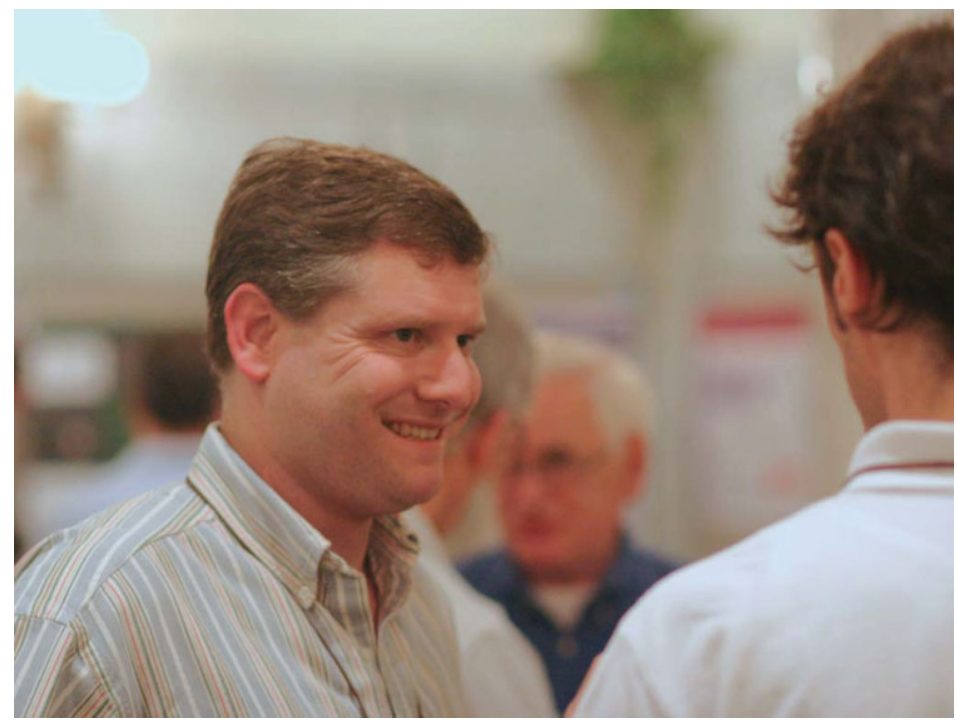

Jay Anderson 